ANNALS OF

CLINICAL

NEUROPHYSIOLOGY

\title{
Associations of nerve conduction study variables with clinical symptom scores in patients with type 2 diabetes
}

\author{
Joong Hyun Park', Jae Hyeon Park', and Jong Chul Won ${ }^{2}$ \\ 'Department of Neurology, Sanggye Paik Hospital, Inje University College of Medicine, Seoul, Korea \\ 2Department of Internal Medicine, Cardiovascular and Metabolic Disease Center, Sanggye Paik Hospital, Inje \\ University College of Medicine, Seoul, Korea
}

Received: November 12, 2018

Revised: December 26, 2018

Accepted: December 28, 2018

\section{Correspondence to}

\section{Jong Chul Won}

Department of Internal Medicine, Cardiovascular and Metabolic Disease Center, Sanggye Paik Hospital, Inje University College of Medicine, 1342 Dongil-ro, Nowon-gu, Seoul 01757, Korea

Tel: +82-2-950-8860

Fax: +82-2-2091-1464

E-mail:drwonjc@gmail.com

\section{ORCID}

Joong Hyun Park

http://orcid.org/0000-0001-6118-4833

Jae Hyeon Park

http://orcid.org/0000-0003-4523-9384

Jong Chul Won

http://orcid.org/0000-0002-2219-4083

Background: Diabetic peripheral polyneuropathy (DPN) is associated with a variety of symptoms. Nerve conduction studies (NCSs) are considered to be the gold standard of nerve damage assessments, but these studies are often dissociated from the subjective symptoms observed in DPN patients. Thus, the aim of the present study was to investigate the correlations between NCS parameters and neuropathic symptoms quantified using the Michigan Neuropathy Screening Instrument (MNSI).

Methods: Patients with type 2 diabetes mellitus (T2DM) with or without symptoms of neuropathy were retrospectively enrolled. Demographic data, clinical laboratory data, MNSI score, and NCS results were collected for analysis; DPN was diagnosed based on the MNSI score ( $\geq 3.0$ ) and abnormal NCS results. Pearson's correlation coefficients were used to evaluate the relationships between MNSI score and NCS variables.

Results: The final analyses included 198 patients (115 men and 83 women) with a mean age of $62.6 \pm 12.7$ years and a mean duration of diabetes of $12.7 \pm 8.4$ years. The mean MNSI score was 2.8 (range, 0.0-9.0), and 69 patients (34.8\%) were diagnosed with DPN. The MNSI score was positively correlated with the median motor nerve latency and negatively correlated with the median motor, ulnar sensory, peroneal, tibial, and sural nerve conduction velocities (NCVs). When the patients were categorized into quartiles according to MNSI score, peroneal nerve conduction velocity was significantly lower in the second MNSI quartile than in the first MNSI quartile ( $p=0.001$ ). A multivariate analysis revealed that the peroneal NCV was independently associated with MNSI score after adjusting for age, sex, and glycosylated hemoglobin Aic (HbA1c) levels.

Conclusions: The present results indicate that a decrease in peroneal NCV was responsible for early sensory deficits in T2DM patients.

Keywords: Diabetic peripheral neuropathy; Nerve conduction study; Michigan Neuropathy Screening Instrument; Peroneal nerve 


\section{INTRODUCTION}

Diabetic peripheral neuropathy (DPN), which is the most common and disabling complication associated with type 2 diabetes mellitus (T2DM), affects up to approximately half of the T2DM patient population. ${ }^{1}$ Recent evidence suggests that DPN begins during the initial stages of $\mathrm{T}^{2} \mathrm{DM}_{;}{ }^{2}$ thus, in terms of both short-term and long-term morbidity, its detection during the early course of diabetes is crucial for a better prognosis and the prevention of subsequent complications, such as diabetic foot ulcers, amputation, and/or disability. ${ }^{3,4}$

Nerve conduction studies (NCSs) are considered the gold standard for the detection of nerve damage ${ }^{5}$ but the results are often dissociated from the subjective symptoms of DPN in patients. ${ }^{6}$ It is not uncommon to encounter diabetic patients with severe neuropathic symptoms but normal NCS parameters because the results of NCSs primarily reflect the function of large myelinated nerve fibers. ${ }^{7}$ However, some studies have shown that the early functional worsening of peripheral nerves in diabetes precedes objective criteria for abnormal NCS findings or even a clinical diagnosis of DPN. ${ }^{8-10}$ A variety of clinical scoring methods have been developed as quantitative instruments to document the presence and severity of DPN. ${ }^{11}$ For example, the Michigan Neuropathy Screening Instrument (MNSI) has been used for the early detection of diabetic neuropathy. ${ }^{12}$ This tool was designed to be used in ambulatory care situations because it can be administered in a relatively short time period (4-5 minutes). Additionally, the MNSI is useful for the evaluation of diabetic neuropathy symptoms and has been validated as a screening test. The specificity of the MNSI for outpatients is $92 \%$, and its reliability and accuracy have been discussed previously. ${ }^{13}$

However, few studies have assessed the relationship between the MNSI score and NCS results. Specifically, no studies have investigated the correlations between these variables using quantitative evaluations of conduction velocity, latency, and amplitude in different nerves. Thus, the aim of the present study was to investigate the correlations between NCS parameters and the neuropathic symptoms quantified using the MNSI.

\section{MATERIALS AND METHODS}

\section{Subjects}

The Research Ethics Committee of Inje University Sanggye Paik Hospital approved the present study (2017-08-025). The requirement for informed consent was waived because the database was accessed only for purposes of analysis; personal information was not used. This study was a retrospective review of the medical records of consecutive T2DM patients who visited the Diabetes Clinic of Inje University Sanggye Paik Hospital for the management of hyperglycemia and an evaluation of complications between January 2014 and December 2016. Of the consecutive T2DM patients who either did or did not exhibit symptoms of neuropathy, those who were examined at least once and had both NCS and the MNSI performed within three weeks of each other were retrospectively enrolled in the present study. The exclusion criteria were as follows: a history consistent with exposure to medications or toxins that could provoke peripheral neuropathy, medical conditions that could potentially develop into peripheral neuropathy (e.g., alcohol consumption, renal failure, hypothyroidism, infections, and/or malignant neoplasms), or suspected carpal tunnel syndrome. Demographic data, previous medical history, vascular risk factors, duration of diabetes mellitus, standard blood tests, NCS variables, and MNSI data were assessed through a retrospective review of medical records. Hypertension was defined as blood pressure $\geq 140 / 90 \mathrm{mmHg}$ or the use of antihypertensive medications, and body mass index (BMI) was calculated as weight in kilograms divided by the square of the height in meters.

\section{Conventional electrophysiological studies}

When possible, the NCS assessment was carried out under conditions in which the patient's skin temperature was above $32^{\circ} \mathrm{C}$. Eligible patients were evaluated using routine procedures, which included motor and sensory NCSs on the unilateral upper and lower extremities with more pronounced neuropathic symptoms. When nerve conduction was not testable on one side, the NCS was performed on the other side. Electromyography was performed using the Sierra Wave EMG system (Cadwell Industries, Kennewick, WA, USA). A single well-trained technician performed all NCS assessments using standard techniques for surface stimulation and recording. ${ }^{14}$ 
The NCSs included measurement of the conduction velocity, latency, and amplitude of the reactions. For the motor conduction studies, an electrical stimulus was delivered to the skin overlying a motor nerve, and the motor responses were recorded from the muscles supplied by each stimulated nerve. For the sensory nerve tests, conduction studies were directly assessed by recording a sensory nerve action potential (SNAPs) proximal to the site of stimulation. The measured NCS parameters included the following variables: compound muscle action potentials (CMAPs); conduction velocities and distal latencies in the median, ulnar, tibial, and peroneal motor nerves; SNAPs; and conduction velocities of the median, ulnar, and sural sensory nerves. The latencies and amplitudes were automatically calculated, and the technician calculated the motor and sensory conduction velocities by measuring the distance between the two stimulating sites and the difference between the latency onsets of the resultant CMAPs. The measured parameters were compared with the normal values used in the laboratories of our research group. The normal ranges of each tested nerve in our laboratories are shown in supplementary Table 1. The NCS results were considered to be abnormal if the latency, amplitude, or velocity of each nerve was not within 2.0 standard deviations from the mean for healthy age-matched controls in two or more separate nerves.

\section{MNSI}

The well-validated Korean version of the self-reported MNSI was used to evaluate the severity of neuropathic symptoms. ${ }^{15}$ The MNSI is a screening and assessment tool for patients with diabetes mellitus who have suspected distal symmetrical peripheral neuropathy. ${ }^{12}$ Of the two separate assessments included in this measure (a 15-item questionnaire and a lower extremity examination), only the 15-item questionnaire was used to compare the NCS parameters with the neuropathic symptoms in the present study. The MNSI consists of items for evaluating foot sensation, including assessments of pain, temperature, tingling, numbness, cramps, and muscle weakness. ${ }^{12}$ The responses are added to obtain a total score with a maximum of 13; 'Yes' responses to items 1-3, 5-6, 8-9, 11-12, and 14-15 and 'No' responses to items 7 and 13 are scored as a single point each; items 4 and 10 (for the measurement of impaired circulation) were not included in the final calculation. A score $\geq 3$ was considered to be abnormal in this study.

\section{Statistical analyses}

All data are expressed as a mean \pm standard deviation (SD). Pearson's correlation coefficients were used to evaluate the relationships between the MNSI score and NCS variables. After categorizing the patients into quartiles according to MNSI score, the differences in the mean values of the NCS variables and prevalence rates of DPN among the four groups were assessed using one-way analysis of variance (ANOVA). Linear regression analyses were performed to confirm the correlation between MNSI score and NCS variables as dependent variables after adjusting for age, sex, and glycosylated hemoglobin (HbA1c) levels. All data were analyzed using SPSS version 23 statistical software (IBM Corp., Armonk, NY, USA). $p<0.05$ were considered to indicate statistical significance.

Table 1. Clinical characteristic of study subjects

\begin{tabular}{lc}
\hline Characteristic & Value \\
\hline Female & $198(41.9)$ \\
Age (years) & $62.6 \pm 12.7$ \\
BMl (kg/m²) & $23.7 \pm 3.3$ \\
\hline Duration of diabetes (years) & $12.7 \pm 8.4$ \\
Hypertension & $99(50.0)$ \\
Retinopathy & $51(25.8)$ \\
\hline HbA1c (\%) & $9.0 \pm 2.7$ \\
FPG (mg/dL) & $202.4 \pm 103.3$ \\
\hline TC (mg/dL) & $167.2 \pm 44.9$ \\
\hline TG (mg/dL) & $150.4 \pm 85.4$ \\
HDL-C (mg/dL) & $45.0 \pm 12.2$ \\
\hline LDL-C (mg/dL) & $110.0 \pm 32.5$ \\
\hline MNSI (score) & $2.8 \pm 2.3$ \\
\hline DPN & $69(34.8)$ \\
\hline Abnormal NCS & $130(65.7)$ \\
\hline Val are exprssed as means
\end{tabular}

Values are expressed as means \pm standard deviation for continuous variables and frequency (\%) for categorical variables.

BMI, body mass index; HbAlc, glycosylated hemoglobin; FPG, fasting plasma glucose; TC, total cholesterol; TG, triglyceride; HDL-C, high density lipoprotein-cholesterol; LDL-C, low density lipoprotein-cholesterol; MNSI, Michigan Neuropathy Screening Instrument; DPN, diabetic peripheral neuropathy; NCS, nerve conduction study. 


\section{RESULTS}

The final analyses in the present study included 198 (115 men and 83 women) of the initial 259 T2DM patients who were referred to the Diabetes Clinic of Sanggye Paik Hospital during the study period. The mean age of the sample was $62.6 \pm 12.7$ years, the mean $\mathrm{HbA} 1 \mathrm{c}$ level was $9.0 \pm 2.7 \%$, the mean duration of diabetes was $12.7 \pm 8.4$ years, and the mean MNSI score was $2.8 \pm 2.3$ (interquartile range [IQR], 1.5-5.4). The NCSs revealed abnormal values in $65.7 \%(n=$ 130) of the sample. When the criteria issued by the Toronto Diabetic Neuropathy Expert Group for DPN were used (MNSI score $\geq 3$ and abnormal NCS), ${ }^{4}$ the prevalence of DPN was $34.8 \%$ (Table 1). Abnormal NCS results were confirmed in $75.3 \%(n=78 / 104)$ of patients with an MNSI score $\geq 3$ ( $n=$ 104), whereas $64.8 \%(n=61 / 94)$ of patients with an MNSI score $<3.0$. The relationships among MNSI score and the NCS variables were assessed with univariate correlation analyses (Table 2) and revealed that the MNSI score was positively correlated with the median motor nerve latency $(p<$ $0.019)$ and negatively correlated with the median motor ( $p=$ $0.005)$, ulnar sensory $(p=0.003)$, peroneal $(p=0.012)$, tibial ( $p$ $=0.024)$, and sural $(p=0.041)$ NCVs

When the patients were categorized into quartiles according to their MNSI score, of the demographic characteristics or laboratory values, only age differed significantly among the four groups. Patients in the second and third MNSI quartiles were significantly older than those in the first and fourth quartiles (Table 3). The percentages of abnormal NCS results in the first, second, third, and fourth MNSI quartiles were $58.1 \%, 70.6 \%, 63.0 \%$, and $69.8 \%$, respectively, but there were no significant differences among the groups. Although the measured NCS parameters did not reach statistical significance, most of parameters, except for distal motor latency, tended to decrease as the MNSI quartiles increased. Table 3 highlights the significant decrease in peroneal nerve conduction velocity in the second MNSI quartile in comparison with the first MNSI quartile $(p=0.001)$. A linear regression analysis revealed that peroneal nerve conduction velocity was independently associated with MNSI score after adjusting for age, sex, and HbA1c level (Table 4).

\section{DISCUSSION}

The present study investigated the relationships among NCS variables and MNSI scores to determine which of the NCS variables were the most sensitive indicators of neuropathic symptom severity. In this study, most of the NCS indices were strongly correlated with the MNSI score, and there was a significant decrease in peroneal NCVs in patients with relatively low symptom scores (first and second MNSI quartiles). The results of this study indicate that the relationship between peroneal NCVs and clinical symptom scores is independent of age, sex, duration of diabetes, and glycemic status (Table 4).

In clinical practice, a DPN diagnosis is typically made using a combination of relevant clinical symptoms and sensory nerve examinations. Recently, a consensus report from the American Academy of Neurology, the joint meeting of the 19th annual Diabetic Neuropathy Study Group of the Euro-

Table 2. Correlation between MNSI score and NCS variable

\begin{tabular}{lcc}
\hline & Pearson's coefficient & $p$-values \\
\hline MMDL & 0.17 & 0.019 \\
MMV & -0.20 & 0.005 \\
MSNAP & -0.01 & 0.913 \\
MSV & -0.13 & 0.071 \\
\hline UMDL & 0.06 & 0.439 \\
UMV & -0.09 & 0.216 \\
USNAP & -0.08 & 0.270 \\
USV & -0.16 & 0.030 \\
PDL & 0.07 & 0.381 \\
PV & -0.19 & 0.012 \\
TDL & 0.11 & 0.128 \\
TV & -0.16 & 0.024 \\
Samp & -0.15 & 0.058 \\
SuV & -0.159 & 0.041 \\
\hline
\end{tabular}

MNSI, Michigan Neuropathy Screening Instrument; NCS, nerve conduction study; MMDL, median motor distal latency; MMV, median motor conduction velocity; MSNAP, median sensory nerve action potential amplitude; MSV, median sensory conduction velocity; UMDL, ulnar motor distal latency; UMV, ulnar motor conduction velocity; USNAP, ulnar sensory nerve action potential amplitude; USV, ulnar sensory conduction velocity; PDL, peroneal distal latency; PV, peroneal conduction velocity; TDL, tibial distal latency; TV, tibial conduction velocity; Samp, sural amplitude; SuV, sural conduction velocity. 
Table 3. Clinical characteristics and NCS variables according to the quartiles of MNSI score

\begin{tabular}{|c|c|c|c|c|c|}
\hline \multirow[b]{2}{*}{ Variable } & \multicolumn{4}{|c|}{ Quartiles of MNSI score } & \multirow[b]{2}{*}{$p$-value for trenc } \\
\hline & $\begin{array}{c}\text { Q1 (0) } \\
(n=43)\end{array}$ & $\begin{array}{l}\text { Q2 (1-2) } \\
(\mathrm{n}=51)\end{array}$ & $\begin{array}{c}\text { Q3 (3-4) } \\
(n=61)\end{array}$ & $\begin{array}{l}\text { Q4 (5-9) } \\
(n=43)\end{array}$ & \\
\hline MNSI score & $0(0)$ & $1(1-1.6)$ & $3(3-3.5)$ & $6(5.8-6.6)$ & $<0.001$ \\
\hline Female & $13(30.2)$ & $22(43.1)$ & $23(37.7)$ & $25(58.1)$ & 0.057 \\
\hline Age (years) & $59.4 \pm 12.8$ & $65.3 \pm 11.0$ & $65.1 \pm 12.8$ & $59.4 \pm 1.3$ & 0.013 \\
\hline BMI $\left(\mathrm{kg} / \mathrm{m}^{2}\right)$ & $23.7 \pm 3.9$ & $23.4 \pm 3.2$ & $24.0 \pm 3.0$ & $23.6 \pm 11.7$ & 0.765 \\
\hline Duration of diabetes (years) & $12.0 \pm 6.6$ & $11.6 \pm 8.3$ & $14.3 \pm 9.2$ & $12.3 \pm 3.3$ & 0.322 \\
\hline Hypertension & $18(41.9)$ & $26(51.0)$ & $31(50.8)$ & $24(55.8)$ & 0.624 \\
\hline Retinopathy & $11(37.9)$ & $13(37.1)$ & $18(43.9)$ & $9(37.5)$ & 0.989 \\
\hline HbAlc (\%) & $8.5 \pm 2.6$ & $8.9 \pm 2.2$ & $9.1 \pm 3.0$ & $9.2 \pm 8.8$ & 0.654 \\
\hline $\mathrm{FPG}(\mathrm{mg} / \mathrm{dL})$ & $149.6 \pm 26.5$ & $175.6 \pm 53.6$ & $210.0 \pm 146.0$ & $284.7 \pm 2.8$ & 0.062 \\
\hline $\mathrm{TC}(\mathrm{mg} / \mathrm{dL})$ & $162.3 \pm 42.2$ & $171.2 \pm 39.0$ & $167.4 \pm 48.7$ & $167.2 \pm 82.9$ & 0.879 \\
\hline $\mathrm{TG}(\mathrm{mg} / \mathrm{dL})$ & $146.9 \pm 86.0$ & $141.0 \pm 98.3$ & $152.5 \pm 79.2$ & $164.1 \pm 48.4$ & 0.741 \\
\hline $\mathrm{HDL}-\mathrm{C}(\mathrm{mg} / \mathrm{dL})$ & $44.1 \pm 11.3$ & $44.2 \pm 11.9$ & $46.2 \pm 14.0$ & $45.2 \pm 75.8$ & 0.882 \\
\hline $\mathrm{LDL}-C(\mathrm{mg} / \mathrm{dL})$ & $102.2 \pm 29.1$ & $108.5 \pm 31.8$ & $113.6 \pm 32.6$ & $114.9 \pm 11.5$ & 0.433 \\
\hline Abnormal NCS & $25(58.1)$ & $36(70.6)$ & $39(63.0)$ & 30 (69.8) & 0.566 \\
\hline DPN & $0(0.0)$ & $0(0.0)$ & $39(63.9)$ & $30(69.8)$ & $<0.001$ \\
\hline MMDL (ms) & $3.6 \pm 0.7$ & $3.7 \pm 0.5$ & $3.8 \pm 0.7$ & $3.9 \pm 0.3$ & 0.168 \\
\hline MMV (m/sec) & $52.2 \pm 4.0$ & $50.4 \pm 5.4$ & $50.7 \pm 4.8$ & $49.3 \pm 8.3$ & 0.074 \\
\hline MSNAP $(\mu \mathrm{V})$ & $15.7 \pm 7.3$ & $15.6 \pm 8.8$ & $16.2 \pm 8.8$ & $16.4 \pm 5.4$ & 0.964 \\
\hline MSV (m/sec) & $43.1 \pm 6.6$ & $40.3 \pm 5.8$ & $41.0 \pm 6.5$ & $40.1 \pm 10.9$ & 0.103 \\
\hline UMDL (ms) & $2.7 \pm 0.4$ & $2.8 \pm 0.5$ & $2.8 \pm 0.5$ & $2.8 \pm 6.5$ & 0.520 \\
\hline UMV (m/sec) & $50.6 \pm 6.6$ & $51.3 \pm 6.1$ & $50.9 \pm 7.2$ & $49.8 \pm 0.4$ & 0.733 \\
\hline USNAP $(\mu \mathrm{V})$ & $13.4 \pm 7.6$ & $12.7 \pm 6.2$ & $12.4 \pm 7.2$ & $12.3 \pm 7.3$ & 0.897 \\
\hline USV (m/sec) & $42.8 \pm 5.1$ & $41.7 \pm 4.8$ & $41.1 \pm 5.2$ & $40.4 \pm 6.2$ & 0.212 \\
\hline PDL (ms) & $3.7 \pm 0.9$ & $4.1 \pm 0.7$ & $4.1 \pm 0.9$ & $3.9 \pm 0.5$ & 0.063 \\
\hline PV (m/sec) & $45.0 \pm 6.3$ & $40.3 \pm 4.8$ & $40.9 \pm 5.6$ & $41.0 \pm 0.7$ & 0.001 \\
\hline TDL (ms) & $3.9 \pm 0.7$ & $4.1 \pm 0.8$ & $4.0 \pm 0.6$ & $4.2 \pm 0.6$ & 0.388 \\
\hline TV (m/sec) & $42.3 \pm 4.9$ & $40.5 \pm 6.3$ & $40.4 \pm 5.3$ & $39.1 \pm 0.6$ & 0.098 \\
\hline Samp $(\mu V)$ & $15.5 \pm 9.0$ & $12.9 \pm 7.1$ & $11.9 \pm 6.2$ & $12.5 \pm 6.6$ & 0.135 \\
\hline $\operatorname{SuV}(\mathrm{m} / \mathrm{sec})$ & $37.2 \pm 5.2$ & $35.7 \pm 3.9$ & $35.2 \pm 4.5$ & $35.2 \pm 7.5$ & 0.203 \\
\hline
\end{tabular}

Values are presented as mean \pm standard deviation or median (interquatile range) for continuous variables and frequency (\%) for categorical variables. Q1/Q2/Q3/Q4, first, second, third, and fourth quartiles, respectively; MNSI, Michigan Neuropathy Screening Instrument; BMI, body mass index; HbA1c, glycosylated hemoglobin; FPG, fasting plasma glucose; TC, total cholesterol; TG, triglyceride; HDL-C, high density lipoprotein-cholesterol; LDL-C, low density lipoprotein-cholesterol; NCS, nerve conduction study; DPN, diabetic peripheral neuropathy; MMDL, median motor distal latency; MMV, median motor conduction velocity; MSNAP, median sensory nerve action potential amplitude; MSV, median sensory conduction velocity; UMDL, ulnar motor distal latency; UMV, ulnar motor conduction velocity; USNAP, ulnar sensory nerve action potential amplitude; USV, ulnar sensory conduction velocity; PDL, peroneal distal latency; PV, peroneal conduction velocity; TDL, tibial distal latency; TV, tibial conduction velocity; Samp, sural amplitude; SuV, sural conduction velocity. 
Table 4. Multivariate analysis for the relationship between clinical and NCS parameters and MNSI score

\begin{tabular}{|c|c|c|c|c|}
\hline & \multicolumn{2}{|c|}{$\begin{array}{l}\text { Unstandardized } \\
\text { coefficients }\end{array}$} & \multirow{2}{*}{$\begin{array}{c}\begin{array}{c}\text { Standardized } \\
\text { coefficients }\end{array} \\
\beta\end{array}$} & \multirow[t]{2}{*}{$p$-value } \\
\hline & $\beta$ & SE & & \\
\hline Constant & 0.654 & 1.918 & & 0.001 \\
\hline Age & -0.017 & 0.014 & -0.096 & 0.223 \\
\hline Sex (female) & 0.490 & 0.333 & 0.111 & 0.143 \\
\hline HbAlc (\%) & 0.022 & 0.069 & 0.026 & 0.745 \\
\hline PV & -0.008 & 0.003 & -0.206 & 0.009 \\
\hline Adjusted $R^{2}$ & 0.08 & & & \\
\hline
\end{tabular}

Adjusted for age, sex, glycosylated hemoglobin and peroneal conduction velocity.

NCS, nerve conduction study; MNSI, Michigan Neuropathy Screening Instrument; SE, standard error; HbA1c, glycosylated hemoglobin; PV, peroneal conduction velocity.

pean Association for the Study of Diabetes (NEURODIAB), and the 8th International Symposium on Diabetic Neuropathy in Toronto (Canada) proposed that a combination of DPN symptom assessments and NCS results yields a more accurate DPN diagnosis. ${ }^{4,5}$ Although NCS is thought to be the gold standard for the assessment of nerve damage, there are often discrepancies between patient symptoms and NCS results in clinical practice. ${ }^{10}$

Findings from the EURODIAB Prospective Complications Study (EPCS), which included 456 patients with type 1 diabetes, showed that peroneal NCVs decrease in patients with neuropathy, nephropathy (both microalbuminuria and macroalbuminuria), and retinopathy (both non-proliferative and proliferative), and that these relationships remain significant after adjusting for age, sex, height, research participant center, diabetes duration, and HbA1c levels. ${ }^{16}$ Additionally, the authors of the study suggested that patients with a clinical suspicion of microvascular complications should be assessed using NCSs to determine the likelihood of subclinical DPN. However, the EPCS was based on a young population of Caucasian Europeans with type 1 diabetes; therefore, the findings may not be generalizable to T2DM patients or other populations. Additional studies investigating the cohort from the Rochester Diabetic Neuropathy Study (RDNS) found that a diagnosis of DPN based on the worsening of NCS variables is superior to a diagnosis made based on clinical symptoms or quantitative sensory nerve tests, even before the criteria for abnormal NCS have been met. Furthermore, the worsening of NCS variables is evident in patients with microalbuminuria and may be an early indicator of microvascular complications. $^{10,17}$

Although it is difficult to compare the findings of the EPCS and RNDS with those of the present study, our study has similar implications in terms of emphasizing the early evaluation of DPN with NCSs. The results of the present study indicate that a significant correlation was found between the MNSI score and NCS variables. Another interesting finding is that the peroneal nerve conduction velocity was significantly lower in patients in the second MNSI quartile than in the first MNSI quartile. Therefore, it is possible that NCSs may be considered when screening for neuropathy, especially in patients with subclinical DPN. Moreover, peroneal NCVs among the NCS indices may be a useful indicator of early nerve damage in T2DM patients.

Previous studies have shown that age, sex, height, duration of diabetes, and glycemic control status $(\mathrm{HbA1c})$ are related to NCS measures. ${ }^{18}$ The Diabetes Control and Complications Trial (DCCT) showed that a poor glycemic control status is associated with deficits in NCS variables, ${ }^{19}$ and the Early Diabetes Intervention Trial found that the duration of diabetes and height are related to the severity of DPN. ${ }^{20}$ However, in the present study, the relationship between the clinical symptom score and peroneal NCV was independent of age, sex, and HbA1c levels. Because the present study did not address this relationship using detailed indices of other microvascular (retinopathy or nephropathy) or macrovascular complications or measures of parameters such as height or BMI, further studies will be necessary to confirm which nerve tests are the most appropriate early and accurate indicators for the evaluation of functional nerve deficits during the development of clinical DPN. Additionally, the present study enrolled patients who were referred from clinics or hospitals for the proper management of uncontrolled diabetes; this may limit the generalizability of the present results. Nonetheless, the prevalence of DPN (34.8\%) in the present study was similar to the rate found in a previous study (33.5\%) that assessed large numbers of patients from 40 secondary or tertiary hospitals in Korea. ${ }^{21}$ Furthermore, the present study showed that $43.9 \%$ of patients with abnormal NCS were subclinical (MNSI < 3), which is comparable to a previous study showing that $44 \%$ of patients with a negative 
Neuropathy Deficit Score had normal sensory and motor signs but abnormal NCS results. ${ }^{22}$ Therefore, the present findings may be representative of actual clinical situations involving T2DM patients.

The pathological and electrophysiological features of nerve damage in DPN include reduced numbers of nerve fibers, decreased conduction amplitudes, and profound decreases in conduction velocities. ${ }^{23,24}$ Additionally, many of these features are typically more prominent in the lower than the upper extremities. Of the nerves that are damaged during the course of DPN, the peroneal nerve is highly vulnerable to these alterations relative to the sural, median, and ulnar nerves. ${ }^{8}$ Therefore, measurements of peroneal NCVs during the early diagnostic window is an effective method for detecting electrophysiological abnormalities in asymptomatic DPN patients. ${ }^{22}$ Consistent with these previous findings, the present correlation analysis revealed that more NCS variables in the lower extremities were prominently related to the clinical symptom score than in the upper extremities (Table 2). Furthermore, although NCVs declined in most of the nerves tested, conduction in the peroneal nerve exhibited a significant decrease even after adjusting for covariates. ${ }^{24}$ Despite the exact mechanisms by which diabetes selectively damages the peroneal nerve remain unknown, the fact that peroneal nerve conduction velocity is the most affected electrophysiological parameter among the NCS parameters suggests that distal sensory motor polyneuropathy due to 'dying back' axonal degeneration is the primary clinical manifestation of DPN.

It has been suggested that neuropathy is an early indicator of microvascular complications. ${ }^{25}$ Currently, NCSs are the most reliable quantitative method available for the objective diagnosis of $\mathrm{DPN}^{4}$ because these tests can identify the slowing of the NCVs, prolongation of latencies, and reductions in nerve action potential amplitudes. For patients with concomitant microvascular complications, a long duration of diabetes, and/or poor diabetes control, NCSs or peroneal NCV assessments may be useful for the detection of nerve damage, even in subclinical cases. Despite recent advances in the understanding of the pathophysiology and natural history of DPN, no breakthroughs regarding the prevention or cure of DPN have been made. ${ }^{23}$ On the other hand, many studies have shown that strict blood glucose control can prevent the progression of DPN and that the early detection of DPN during regular follow-up visits can be achieved with appropriate screening and accurate diagnostic tests. ${ }^{11}$

In conclusion, the present study's finding of a considerable correlation between the MNSI score and NCVs may improve physicians' understanding of the clinical course and neuropathic symptoms of patients with T2DM. Furthermore, of the abnormal NCS parameters identified, decreases in peroneal NCVs might be indicative of early sensory deficits in T2DM patients. Thus, the results support a more stratified or personalized management approach to T2DM patients.

\section{Conflicts of Interest}

No potential conflict of interest relevant to this article was reported.

\section{Acknowledgment}

This work was supported by the grant from the Priority Research Centers Program and Basic Science Research Program through the National Research Foundation of Korea funded by the Ministry of Education, Science, and Technology (NRF2010-0020224, NRF-2015R1A2A1A13001900).

\section{REFERENCES}

1. Tesfaye S, Selvarajah D. Advances in the epidemiology, pathogenesis and management of diabetic peripheral neuropathy. Diabetes Metab Res Rev 2012;28 Suppl 1:8-14.

2. Malik RA, Tesfaye S, Newrick PG, Walker D, Rajbhandari SM, Siddique I, et al. Sural nerve pathology in diabetic patients with minimal but progressive neuropathy. Diabetologia 2005;48:578585.

3. Caputo GM, Cavanagh PR, Ulbrecht JS, Gibbons GW, Karchmer AW. Assessment and management of foot disease in patients with diabetes. N Engl J Med 1994;331:854-860.

4. Tesfaye S, Boulton AJ, Dyck PJ, Freeman R, Horowitz M, Kempler $P$, et al. Diabetic neuropathies: update on definitions, diagnostic criteria, estimation of severity, and treatments. Diabetes Care 2010;33:2285-2293.

5. England JD, Gronseth GS, Franklin G, Miller RG, Asbury AK, Carter GT, et al. Distal symmetric polyneuropathy: a definition for clinical research: report of the American Academy of Neurology, the American Association of Electrodiagnostic Medicine, and the American Academy of Physical Medicine and Rehabilitation. 
Neurology 2005;64:199-207.

6. Pastore C, Izura V, Geijo-Barrientos E, Dominguez JR. A comparison of electrophysiological tests for the early diagnosis of diabetic neuropathy. Muscle Nerve 1999;22:1667-1673.

7. Malik RA, Veves A, Tesfaye S, Smith G, Cameron N, Zochodne D, et al. Small fibre neuropathy: role in the diagnosis of diabetic sensorimotor polyneuropathy. Diabetes Metab Res Rev 2011;27:678684.

8. Dyck PJ, Karnes UL, Daube J, O'Brien P, Service FJ. Clinical and neuropathological criteria for the diagnosis and staging of diabetic polyneuropathy. Brain 1985;108(Pt 4):861-880.

9. Dyck PJ, Karnes JL, O'Brien PC, Litchy WJ, Low PA, Melton $L J$ 3rd. The Rochester Diabetic Neuropathy Study: reassessment of tests and criteria for diagnosis and staged severity. Neurology 1992:42:1164-1170.

10. Dyck PJ, O'Brien PC, Litchy WJ, Harper CM, Klein CJ. Monotonicity of nerve tests in diabetes: subclinical nerve dysfunction precedes diagnosis of polyneuropathy. Diabetes Care 2005;28:2192-2200.

11. Perkins BA, Olaleye D, Zinman B, Bril V. Simple screening tests for peripheral neuropathy in the diabetes clinic. Diabetes Care 2001;24:250-256.

12. Feldman EL, Stevens MJ, Thomas PK, Brown MB, Canal N, Greene DA. A practical two-step quantitative clinical and electrophysiological assessment for the diagnosis and staging of diabetic neuropathy. Diabetes Care 1994;17:1281-1289.

13. Lunetta M, Le Moli R, Grasso G, Sangiorgio L. A simplified diagnostic test for ambulatory screening of peripheral diabetic neuropathy. Diabetes Res Clin Pract 1998;39:165-172.

14. Johnsen B, Fuglsang-Frederiksen A. Electrodiagnosis of polyneuropathy. Neurophysiol Clin 2000;30:339-351.

15. Kim SS, Won JC, Kwon HS, Kim CH, Lee JH, Park TS, et al. Prevalence and clinical implications of painful diabetic peripheral neuropathy in type 2 diabetes: results from a nationwide hospital-based study of diabetic neuropathy in Korea. Diabetes Res Clin Pract 2014;103:522-529.

16. Charles M, Soedamah-Muthu SS, Tesfaye S, Fuller JH, Arezzo JC, Chaturvedi $\mathrm{N}$, et al. Low peripheral nerve conduction velocities and amplitudes are strongly related to diabetic microvascular complications in type 1 diabetes: the EURODIAB Prospective Complications Study. Diabetes Care 2010;33:2648-2653.

17. Mauer SM, Goetz FC, McHugh LE, Sutherland DE, Barbosa J, Najarian JS, et al. Long-term study of normal kidneys transplanted into patients with type I diabetes. Diabetes 1989;38:516-523.

18. Ibers JW, Herman WH, Pop-Busui R, Martin CL, Cleary P, Waberski B. Subclinical neuropathy among Diabetes Control and Complications Trial participants without diagnosable neuropathy at trial completion: possible predictors of incident neuropathy? Diabetes Care 2007;30:2613-2618.

19. Diabetes Control and Complications Trial (DCCT): results of feasibility study. The DCCT Research Group. Diabetes Care 1987;10:119.

20. Tkac I, Bril V. Glycemic control is related to the electrophysiologic severity of diabetic peripheral sensorimotor polyneuropathy. Diabetes Care 1998;21:1749-1752.

21. Won JC, Kwon HS, Kim CH, Lee JH, Park TS, Ko KS, et al. Prevalence and clinical characteristics of diabetic peripheral neuropathy in hospital patients with type 2 diabetes in Korea. Diabet Med 2012;29:e290-e296.

22. Baba M, Ozaki I. Electrophysiological changes in diabetic neuropathy: from subclinical alterations to disabling abnormalities. Arch Physiol Biochem 2001;109:234-240.

23. Dyck PJ, Karnes JL, O'Brien P, Okazaki H, Lais A, Engelstad J. The spatial distribution of fiber loss in diabetic polyneuropathy suggests ischemia. Ann Neurol 1986;19:440-449.

24. Albers JW, Brown MB, Sima AA, Greene DA. Nerve conduction measures in mild diabetic neuropathy in the Early Diabetes Intervention Trial: the effects of age, sex, type of diabetes, disease duration, and anthropometric factors. Tolrestat Study Group for the Early Diabetes Intervention Trial. Neurology 1996;46:85-91.

25. Binns-Hall O, Selvarajah D, Sanger D, Walker J, Scott A, Tesfaye S. One-stop microvascular screening service: an effective model for the early detection of diabetic peripheral neuropathy and the high-risk foot. Diabet Med 2018;35:887-894. 